\title{
DDCA in Cellular Network using Fuzzy Rule Based Multi- agent System
}

\author{
Megha Kamble \\ Research Scholar \\ R.G.P.V. \\ Bhopal
}

\author{
Dr.Roopam Gupta \\ H.O.D., Dept .of I.T. \\ R.G.P.V. \\ Bhopal
}

\begin{abstract}
Present mobile networks have different Quality of Service (QoS) requirements to support increasing need with major objectives such as better admission control, effective bandwidth utilization, and fault tolerance.

So the network aims at satisfying their demands without much degradation of quality in terms of call dropping and call blocking. It is possible by admission control algorithms and by optimizing the sharing and utilization of existing resources in an effective way. It improves the overall performance of the network system. This paper addresses different Quality of Service (QoS) requirements of the users of a cellular network with a focus on fuzzy based Call Admission Control Scheme (CAC) and optimized bandwidth allocation using Multi Agent System Model. We propose combined framework to address resource allocation problem. This problem can be dealt with the help of two issues in Admission control and Transmission control. The admission control - the decision to admit or reject the calls based on fuzzy logic control. Transmission control is provided thru Distributed Dynamic Channel allocation(DDCA) using co-operative negotiation of Multi-Agent System. We demonstrate the efficiency of the proposed framework model by determining call dropping probability and call blocking probability.
\end{abstract}

\section{Categories and Subject Descriptors}

I[Computing Methodologies]: I.1.2 Algorithms, Analysis of algorithm, I.2 Artificial Intelligence, Fuzzy Logic

\section{General Terms}

Algorithms

\section{Keywords}

Multi-agent systems, Channel Allocation, Fuzzy rules for Call Admission, Load balancing, Cellular Sy stem, Negotiation

\section{INTRODUCTION}

In cellular mobile network, the geographical area is divided into hexagonal cells. The mobile hosts in each cell are serviced by a base station (BS) located in the cell and the BSs are interconnected via a wired network. The available wireless bandwidth is divided into channels, where each channel is capable of supporting a communication session. Cellular Network is having scarce radio resources and no. of users are increasing every day. The tremendous growth of usage of mobile network has increased the need for better admission control strategies and efficient utilization of resources such as bandwidth, to provide a better Quality of Service (QoS). Call Admission Control (CAC) is a fundamental mechanism and channel allocation is a key problem in cellular network.

In the literature, call admission control is handled by different mechanism. The admission control for mobile networks based on thresholds and network status are popular. Another Admission Control method by the authors of [4] is performed considering two levels. The new calls are blocked by the admission control if either the number of calls exceeds the limit value of resources allocated. In this case, handover calls are rejected as well as the new calls. Call admission control is thus done at the level of the configuration of domain bandwidth. The authors of [5] have suggested call admission by rearrangements of the calls already in progress. When the offered traffic load is heavy, i.e., the sum of the requested bandwidth exceeds the available bandwidth so that not all the requests can be completely served, and the user mobility is high, some form of call admission control needs to be employed. The online call admission strategy is based on bandwidth reservation. This gives priority to handoff calls over new calls. This means that, in a heavy traffic load situation, low priority calls are almost never allocated outer layer bandwidth. In the case of a handoff call, it is accepted if there is available (reserved or unused) outer layer bandwidth to accept a requested handoff call. The limitation of the techniques can be eliminated by a fuzzy logic based admission control method that involves less computational efforts and also allows consideration of the system behavior.

There are different strategies for channel allocation in Cellular Networks such as fixed channel allocation and dynamic channel allocation. This is implemented using centralized approach or distributed approach[8][9]. This paper implements distributed dynamic channel allocation(DDCA) strategy using a multi-agent system virtually ported at Base station[2][3]. The agents here are software agents, represent network operators that manage the radio resource of different regions of the cellular network. The extra dimension in a wireless network is the allocation of channel to a cell to avoid interference and avoid degradation in QoS. 
Bodanese[3] has worked for distributed resource allocation scheme for first generation mobile networks using intelligent agents. This scheme of resource allocation worked under moderate to heavy loads and has shown several advantages. John Bigham, Lin $\mathrm{Du}[10]$ had introduced co-operative negotiation process where co-operative layer executes a join plan by broadcasting request to all the co-channel cells and negotiating with the cell which is ready for agreement. Recently Panagiotis Minas Papazoglou, Dimitrios Alexios Karras[1] have presented improved multi-agent system, which balanced the load of network using thread delay based negotiation and network performance based negotiation.

All the strategies discussed above deals either with CAC or resource allocation separately. But for QoS requirement both the issues are to be considered critically. This paper proposes a combined framework - Fuzzy rules for CAC and resource allocation using multi-agent system model. MAS concentrates on channel allocation using chained negotiation and tries to increase decision making of individual base stations in the mobile network in load balancing of the network. This approach will also reduce call dropping probability and call blocking probability.

The rest of the paper is organized as follows. In section 2, we introduce functional architecture of Multi-Agent System designed to handle channel assignment, and propose a negotiation process in Section 3. Fuzzy rules for CAC will also be discussed in section 2. The simulation experiment parameters and results are discussed in section 4 . We conclude our paper with a summary in section 5 .

\section{FUNCTIONAL ARCHITECTURE}

A multi-agent system (MAS) can be defined as a group of agents. The agents interact with the environment and with each other in a co-coordinated way. In this work, we have adapted hybrid architecture (combining Logical and Reactive model) of MAS known as Integration of Reactive behavior and Rational Planning architecture (INTERRAP) architecture[3]. Each agent has a multilayered architecture and every layer is designed with specific functionality. In this work, every agent is an intelligent agent, and MAS has a layered architecture with both planning and reactive components.

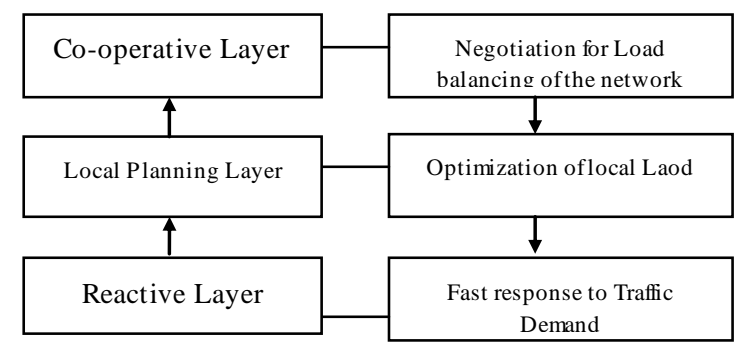

Figure 1 Layered Architecture of an Agent

The reactive layer is based on Dy namic Channel Allocation. When a new call is originated by a mobile host in the same cell, reactive layer stores a channel from central spectrum and allocates it to the call. When a hand off call is transferred from neighboring region, reactive layer checks the free channel in local set of available channels and allocates it to the hand-off call. But when there is no free channel available in the local set, request is transferred to Local Planning Layer. Local Planning Layers is allocating channel to the request using reassignment scheme and borrowing channels from co-channel cells [2].

Software agent in Local planning layer calculates degree of coldness $-\operatorname{deg}_{\mathrm{c}}$ (ratio between no. of available channels and no. of channels allotted to cell at a particular time instant)[4]. Local Planning layer is maintaining a threshold value $h$, for degree of coldness $-\operatorname{deg}_{c}$. If $\operatorname{deg}_{c}<h$, and scenario remains same for certain amount of time[4], intelligent software agent of Local Planning lay er will trigger an event for free channel which is coordinated by co-operative layer. Co-operative layer plays the role for overall performance of the cellular network and so starts negotiation process.

The results of this work will allow the development of a new channel allocation algorithm for cellular networks that intelligently changes traffic distribution in real time using negotiation and will be helpful for increased channel utilization. The autonomy given to base station will introduce an increase in flexibility to deal with new situations in traffic load, and to decrease the call dropping probability of the network.

\subsection{Fuzzy Logic for Admission Control}

In this Multi-Agent model, reactive layer will take the initiative for admission control that is allowing admission to call whether it is a new call or call in progress. . It is generally accepted that it is less desirable to drop a call in progress than to reject a new call, and as a consequence, the Admission Control algorithms for cellular networks have two target parameters: the call dropping probability and the call blocking probability. This paper determines the probability of call admission using fuzzy rules instead of finding exact parameter value. Every time reactive layer determines the traffic load. Traffic Load is no. of calls arrived per hr. and can also be expressed in Erlangs.

The following simple fuzzy rules decide whether to accept a call or reject the call[6].

1) If Traffic Load is very low, then admission decision is Strongly Accept (SR).

2) If Traffic Load is low then admission decision is Strongly Accept (WA)

3) If Traffic Load is average, then admission decision is Weakly Accept with Buffering(WAB).

4) If Traffic Load is above average, then admission decision is Weakly Reject with Buffering(WRB).

5) If Traffic Load is high, then admission decision is Weakly Reject with Buffering(WRB). 
6) If Traffic Load is very high then admission decision is Strongly Reject.(SR).

The strongly rejected calls will be dropped, but rest of the calls will be admitted sometimes with delay and this will reduce call dropping probability.

\section{COOPERATIVE NEGOTIATION FOR DDCA IN A MULTI AGENT SYSTEM}

\subsection{Problem domain}

One of the basic principles of cellular networks is the reuse of radio resources beyond a certain distance (min. reuse distance). All the cells reusing same set of channels are known as co-channel cells. Heavy traffic cells can borrow channels from their cochannel cells. Such uneven traffic distribution and demand gives rise to hot regions and cold regions. So channel allocation strategy should be distributed in nature. This work proposes distributed channel allocation and dynamism is handled with the help of agent interaction in MAS.

When the cell is becoming heavily loaded, it's co-operative layer starts negotiation process and tries to execute joint plan with neighbors. Negotiation can be defined as a process of solving the resource allocation problem through the structured exchange of messages. Negotiation process is part of communication and communication can be triggered by an agent on user interaction or by the agent automatically (using Intelligent decision making). There is considerable research on negotiation that has been carried out in the domains of Artificial Intelligence and so especially it is used in real-time systems like cellular network.

In this work, negotiation is used as an intelligent control technique to allot channel from cold cell to hot cell dy namically and balance the traffic load over the whole cellular network. This work uses negotiation for execution of management handoffs. The cooperative coverage negotiation is triggered when the local traffic in any cell exceeds a certain threshold, as the heavily loaded base station has to shrink its coverage to reduce its utilization and negotiate with others for covering its coverage loss. Negotiation process introduced in this paper is known as Chained negotiation. This will overcome the problem of link failure or base station failure[2], thus support fault tolerance and every cell agent doesn't have to wait for all the replies.

Co-operative layer works in three steps for chained negotiation:

1) Identify all the active co-channel cells. Ask nearest neighbor co-channel cell[3] for degree of coldness. If $\operatorname{deg}_{c}<h$, request the co-operative layer for Average Channel Occupancy ACO. ACO is calculated as

$\mathrm{ACO}=($ ratio of no. of channels in use to total no. of channels allotted/borrowed by cell) for $\mathrm{N}$ no. of calls. Initially co-operative layer of a base station will assume some value for ACO. Let's call it as TACO(threshold for Average Channel Occupancy). If difference of TACO \& ACO is a greater than value of approximate call arrival time, this neighboring co-channel cell will accept joint plan.

The process further explained with the help of an example.

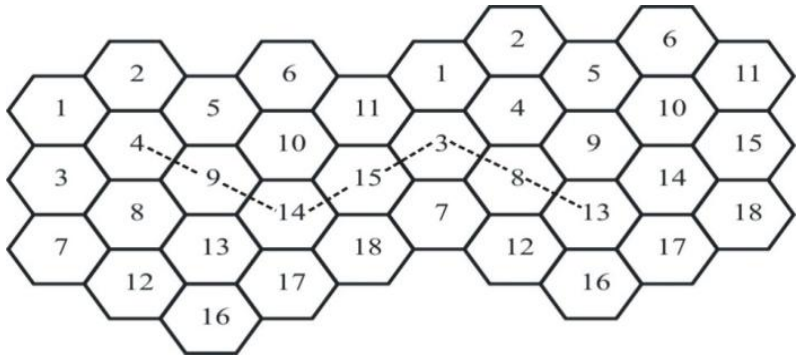

Figure 2 Hexagonal Model of Cellular Network \& Chained Negotiation

Refer to Fig.2, This is hexagonal Cellular network wrap around model, based on 7-cluster structure. So any Cell is having 6 cochannel cells. Suppose at time instant t, Cell no. 4 becomes hot and co-operative layer of the cell initiates negotiation. C-operative layer will communicate request for joint plan to a co-channel cell, say Cell no. 14. Cell 14 is not having any free channel. So Cell no. 14 will become initiator of negotiation and communicate to cochannel cells except Cell No. 4. This will reduce negotiation tenure if correct alternative is chosen in the first step.

Optimal solution will be find out using heuristics. The heuristic will be degree of coldness and average channel occupancy. To avoid indefinite chaining, hop count is measured and maintained upto a threshold limit. Threshold limit will be cluster size -2 .

2) Identify the calls to be transferred - . When it is not possible for reactive layer to allocate a channel to a call, some calls can be moved from hot cell to cold sell and this is known as management handoff. But the constraint for management handoff is only mobile hosts close to certain borders of the cells can be moved from one cell to other.

Calls are categorized as New calls, Hand-off calls and departing calls based on call duration. New calls are given the priority for management handoffs.

\subsection{Negotiation Process}

Negotiation process is a part of negotiation system[10]. The system is consisting of

1) The negotiation protocols are the set of rules that govern the interaction. This covers the participants and types of messages.

for ex.. Request for degree of coldness

Request for average channel occupancy

Accepting the request and acknowledgement for joint plan

Request for less frequently used Channel

Transfer of channel 
2) The negotiation states : initiation, partial completion, rejection, finished

3) Negotiation resolution: After comparing the parameters joint plan may be executed or terminated by both the agents.

The main objective of negotiation process of co-operative layer of every cell is to acquire a free channel for call establishment when dy namically require. That objective should be achieved in a certain amount of time and with less message complexity. Outcome of the negotiation process can be evaluated for two parameters:

The Traffic blocking rate is one of the most important characteristics for measuring the performance of a cellular network. When a new call originates and the network can not allocate a channel then, we say that this call is blocked.

$$
\text { Blocking rate }=\frac{\text { no. of blocked calls }}{\text { no. of total calls }}
$$

Eq.(1)

The handoff rejection rate is also an additional and very important characteristic for measuring the cellular network performance. When a call is in progress and the required quality conditions are not met then, this call is obligatorily driven to termination.

Eq.(2)

Handoff Rejection Rate $=\frac{\text { no. of calls dropped }}{\text { no. of total calls - no. of blocked calls }}$

\section{SIMULATION RESULTS}

\subsection{Simulation Results}

In this work, we do not consider users mobility and their precedence. Omnet++ is the simulator used for result analysis. 3cell cluster model is used. The results are generated by changing the number of channels and monitoring the simulation for specific demand.

Table 1. Call dropping probability calculated by varying traffic load

\begin{tabular}{|c|c|c|c|}
\hline Sr.No & $\begin{array}{c}\text { Total no. of } \\
\text { channels } \\
\text { available }\end{array}$ & $\begin{array}{c}\text { Traffic } \\
\text { Load } \\
\text { calls/hr }\end{array}$ & $\begin{array}{c}\text { Call Dropping } \\
\text { Probability(\%) }\end{array}$ \\
\hline 1 & 30 & 35 & 0 \\
\hline 2 & 30 & 60 & 0.37 \\
\hline 3 & 45 & 90 & 0.4 \\
\hline 4 & 60 & 90 & 0.12 \\
\hline
\end{tabular}

\section{CONCLUSION}

This work describes a new combination of Multi- agent system and Fuzzy rule based admission control algorithm for Cellular Network. We have shown reduced call dropping probability in a simple simulation scenario.

Our future work will use the result from this paper in order to improve and extend the fuzzy logic for channel utilization.

\section{REFERENCES}

[1] Panagiotis Minas Papazoglou, Dimitrios Alexios Karras, "An Improved Multi-Agent Simulation Methodology for Modelling and Evaluating Wireless Communication Systems Resource Allocation Algorithms", ISSN 10043756/05/1402/129 Journal Of Systems Science And Systems Engineering, Oct 2008.

[2] Jaspreet Singh, Jaspreet Kaur, Sandeep Pal Singh, “Algorithms for Distributed Dynamic Channel Allocation”, National Conference on RAFIT 2005 2nd-3rd March, 2005

[3] E.L. Bodanese And L.G. Cuthbert, “Application Of Intelligent Agents In Channel Allocation Strategies For Mobile Networks", IEEE International Conference On Communications, Vol. 1, Pp. 18-22, June 2000

[4] Guohong Cao, Associate Member, IEEE, And Mukesh Singhal, Senior Member, IEEE, "Distributed Fault-tolerant Channel Allocation For Cellular Networks", Ieee Journal On Selected Areas In Communications, Vol. 18, No. 7, JULY 2000 Pp. 1326-1337

[5] Jianchang Yang, D. Manivannan, “ An Efficient Fault-tolerant Distributed Channel Allocation Algorithm For Cellular Networks", IEEE Transactions On Mobile Computing, VOL. 4, No. 6, November/December 2005

[6] D. Todinca, “Applying Fuzzy Logic to Admission Control in GPRS/EGPRS Networks", Periodica olitehnica, Transactions on Automatic Control and Computer Science, vol. 48 (62), pp. 56-61, 2003.

[7] Sonia Ben Rejeb, Zièd Choukair, Sami Tabbane, "Resource Allocation and QoS Management for Cellular Multimedia Network", GRES February 2003

[8] I. Katzela and M. Naghshineh, "Channel Assignment Schemes for Cellular Mobile Telecommunication Systems: A Comprehensive Survey", IEEE Personal Communications Mag., vol. 3, no. 3, pp 10-31, June 1996.

[9] Prakash R., Shivaratri, N.G., Singhal, M.: "Distributed Dynamic Fault-Tolerant Channel Allocation for Mobile Computing," IEEE Transactions on Vehicular Technology,vol.48(6), November 1999, pp.1874-1888.

[10] John Bigham, Lin Du, “ Cooperative Negotiation in a MultiAgent System for RealTime Load Balancing of a Mobile Cellular Network", AAMAS'03, July 14-18, 2003, Melbourne, Australia.

[11] Nupur Giri, Shrikant Bodhe, "Performance Evaluation of Multi Agent Based Call Admission in Cellular Networks", International Journal of Recent Trends in Engineering, Vol 1, No. 1, May 2009 